\title{
ESTRUTURA E COMPOSIÇÃO FLORÍSTICA DO ESTRATO ARBÓREO EM DUAS ZONAS ALTITUDINAIS NA MATA ATLÂNTICA DE ENCOSTA DA REGIÃO DO IMBÉ, RJ' ${ }^{1}$
}

\author{
Marcel R. Moreno ${ }^{2}$ \\ Marcelo T. Nascimento ${ }^{2}$ \\ Bruno C. Kurtz ${ }^{3}$
}

Recebido em 25/03/2002. Aceito em 17/12/2002

\begin{abstract}
RESUMO - (Estrutura e composição florística do estrato arbóreo em duas zonas altitudinais na Mata Atlântica de encosta da região do Imbé, RJ). O objetivo deste estudo foi avaliar a composição florística e estrutural do estrato arbóreo de um remanescente de mata atlântica submontana na Região do Imbé, RJ, testando a hipótese de que diferenças em altitude (zona 1 localizada a $50 \mathrm{~m} . \mathrm{s} . \mathrm{m}$. e zona 2 localizada a $250 \mathrm{~m} . \mathrm{s} . \mathrm{m}$.) acarretam alterações na comunidade. O total de dez parcelas de 30×40m foram alocadas em duas zonas altitudinais, cinco no sítio 1 e outras cinco no sítio 2. Todas as árvores com DAP $\geq 10 \mathrm{~cm}$ foram marcadas e medidas. Dentre os 940 indivíduos amostrados, um total de 210 espécies foram identificadas, distribuídas em 158 gêneros e 43 famílias. As duas zonas estudadas apresentaram valores semelhantes de densidade total, área basal total e diversidade de espécies. Os valores de diversidade de espécies estão entre os mais altos encontrados em Mata Atlântica. A família Euphorbiaceae apresentou os maiores valores de $\mathrm{VC}$ em ambas as zonas altitudinais. A família mais rica em número de espécies foi Leguminosae (26 espécies). Das 210 espécies amostradas, apenas 57 foram comuns às duas zonas amostradas. Na zona 1, as espécies com os maiores VC foram Hyeronima alchorneoides Allemão (14,3), Actimostemon verticilatus (K1.) Baill. $(11,4)$ e Rustia formosa (Cham. \& Schltal.) Klotzch $(10,7)$, enquanto que para a zona 2, foram Actimostemon verticilatus $(13,8)$, Euterpe edulis Mart. (12,4) e Mabea fistulifera Mart. (8,1). Os resultados indicaram que a composição florística mudou em função da altitude, mas não a estrutura da floresta e a diversidade de espécies.
\end{abstract}

Palavras-chave - fitossociologia, florística, diversidade de espécies, Mata Atlântica

\begin{abstract}
Structure and floristic composition of tree communities in two altitudinal zones in an Atlantic forest in the Imbé Region, RJ, Brazil). The Imbé is one of the main Atlantic forest remnants in the Rio de Janeiro State. The aim of this study was to test whether differences in forest structure and floristic composition exist between two tree communities at different altitudes (site 1 at $50 \mathrm{~m}$ high and site 2 at $250 \mathrm{~m}$ high). Five plots of $30 \mathrm{~m} \times 40 \mathrm{~m}\left(1200 \mathrm{~m}^{2}\right)$ were setup in each site. All trees with DBH $\geq 10 \mathrm{~cm}$ were tagged and measured. A total of 210 species was sampled, distributed in 158 genus and 43 families. Both sites is showed similar values for basal area, number of individuals and canopy height. The species diversity $\left(H^{\prime}=4,21\right.$ and 4,30, respectively to site 1 and site 2$)$ can be considered to be within the highest values found for Atlantic forests. Among the families, the Euphorbiaceae

1 Parte da dissertação de Mestrado do primeiro Autor

2 Laboratório de Ciências Ambientais, CBB, Universidade Estadual do Norte Fluminense (UENF), Campos dos Goytacazes, CEP 28013-600, Rio de Janeiro, RJ, Brasil

3 Instituto de Pesquisas Jardim Botânico do Rio de Janeiro, Rua Pacheco Leão 915, CEP 22460-030, Rio de Janeiro, RJ, Brasil
\end{abstract}


had the highest values of CV in both altitudinal sites. The family with the highest richness of species was the Leguminosae ( 26 species). Only 57 species were common to both forest sites. At site 1 , the species with the highest CV were Hyeronima alchorneoides Allemão $(14,3)$, Actimostemon verticilatus $(11,4)$ and Rustia formosa $($ Cham. \& Schltal.) Klotzch (10,7), while for the forest at site 2 they were Actimostemon verticilatus (K1.) Baill. (13,8), Euterpe edulis Mart. $(12,4)$ and Mabea fistulifera Mart. $(8,1)$. The results showed that the floristic composition changed with altitude, but not the forest structure and species diversity.

Key words - phytosociology, Floristic Atlantic Forest, species diversity, Brazil

\section{Introdução}

As áreas de Mata Atlântica ainda hoje bem preservadas estão localizadas basicamente em escarpas muito íngremes ou em altitudes elevadas, onde a prática agrícola ou madeireira se torna inviável, além de outras poucas áreas de preservação ambiental. $\mathrm{O}$ desmatamento tem ocorrido de forma mais intensa na região compreendida entre o norte do Estado do Rio de Janeiro e o sul da Bahia (Fundação SOS Mata Atlântica 1998). A maioria das áreas no Estado do Rio de Janeiro foi ou tem sido utilizada para atividades agropecuárias, em especial o plantio de café, cana-de-açúcar e pastagens.

Para a Mata Atlântica no Estado do Rio de Janeiro e, em especial, para a região Norte Fluminense, estudos fitossociológicos são reduzidos (Pessoa et al. 1997; Guedes-Bruni et al. 1997; Moreno et al. 1998; Kurtz \& Araújo 2000; Silva \& Nascimento 2001) sendo a maioria de caráter descritivo, não havendo abordagem da relação vegetação-ambiente.

O papel da altitude na composição florística e na estrutura das florestas tropicais tem sido demonstrado por vários autores (Proctor et al. 1988; Liberman et al. 1995; 1996; Pendry \& Proctor 1996) sendo que, ao longo de um gradiente altitudinal, muitos fatores do complexo ambiental, tais como temperatura, precipitação pluviométrica, umidade, velocidade dos ventos e outros, mudam concomitantemente. A topografia também influencia os distúrbios no sistema, por aumentar a susceptibilidade do sítio a determinados tipos de problemas, tais como deslizamentos (Hadley 1994).
Este trabalho teve por objetivos: 1) avaliar a composição florística e a estrutura do estrato arbóreo em dois trechos da floresta submontana (50 e 250m.s.m.) da região do Imbé, RJ, testando a hipótese de que diferenças em altitude acarretam alterações na composição e na estrutura da comunidade; e 2) estabelecer uma coleção de referência para a região, a ser depositada no herbário da UENF (em fase de implantação).

\section{Material e métodos}

Área de estudo - A região do Imbé (Fig. 1) está localizada no município de Campos dos Goytacazes ( $21^{\circ} 48^{\prime} \mathrm{S}, 14^{\circ} 40^{\prime} \mathrm{W}$ ), fazendo parte do Parque Estadual do Desengano e de seu entorno, que representam um dos maiores remanescentes de floresta ombrófila densa atlântica, que outrora ocupou boa parte da área do Estado do Rio de Janeiro. Esta floresta encontra-se hoje ilhada e fragmentada por uma série de atividades agropecuárias que se desenvolvem na região desde a colonização, principalmente pelo plantio de cana-de-açúcar e pastagem.

Este estudo foi desenvolvido na vertente atlântica da Serra do Imbé que é recoberta por Floresta Ombrófila Densa Submontana (Radambrasil 1983). A região possui uma série de pequenos rios que têm origem geralmente nas regiões altas das montanhas e apresentam grande importância econômica e ecológica.

Os solos são relativamente rasos, com grande número de rochas aflorando, em especial na cota de $250 \mathrm{~m} . \mathrm{s} . \mathrm{m}$., variando de franco-arenosos (mata a 50m.s.m.) a franco argilo-arenosos (mata a $250 \mathrm{~m} . \mathrm{s} . \mathrm{m}$.), sendo o 


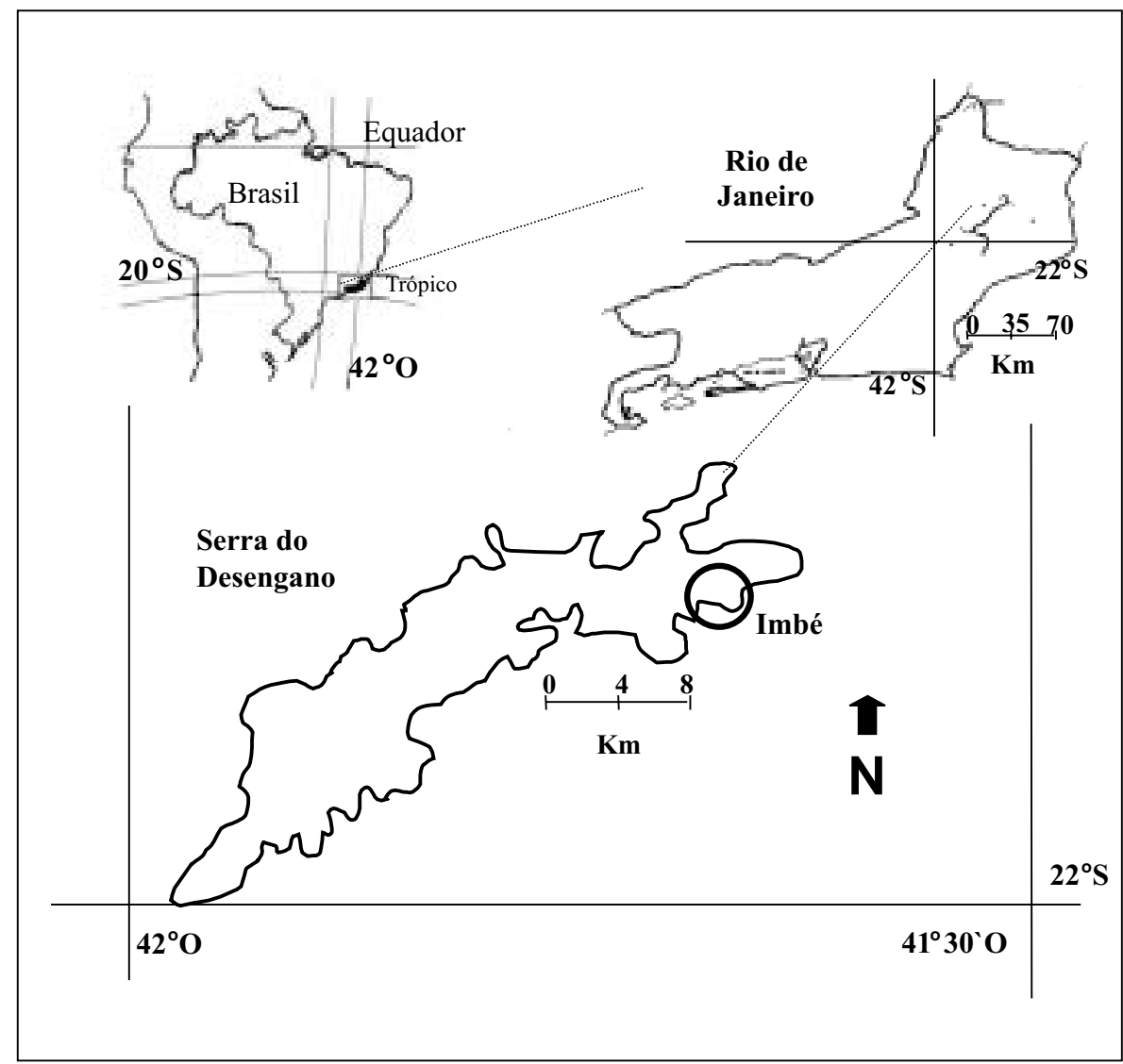

Figura 1. Mapa com a localização da Região do Imbé, RJ.

solo desta última, em geral, mais rico em nutrientes (Mazurec 1998). O relevo é acidentado e variável, alternando algumas áreas planas com outras de declive bastante acentuado.

O clima local se mostra nitidamente estacional, com inverno seco e verão úmido, principalmente nas regiões mais baixas. Com o aumento da altitude, o efeito orográfico diminui o caráter estacional do clima. As precipitações médias anuais ficam em torno de $1.300 \mathrm{~mm}$. A temperatura média anual varia de acordo com a altitude, sendo maior que $19^{\circ} \mathrm{C}$, entre 0 e $200 \mathrm{~m}$, e entre 17 e $25^{\circ} \mathrm{C}$, nas altitudes situadas entre $200 \mathrm{e}$ $500 \mathrm{~m}$. Na vertente atlântica, o clima predominante é o úmido, com pouco ou nenhum déficit de água, e mesotérmico, com temperaturas elevadas bem distribuídas o ano todo (Radambrasil 1983).

No século XIX a mata Atlântica no Rio de Janeiro, principalmente nas áreas altas como a região do Imbé, foi em grande parte substituída pelas plantações de café (Dean 1997), sendo possível encontrar ainda hoje pés de café no sub-bosque de algumas matas da região. Este autor também descreve como outra importante atividade antrópica na região, principalmente até 1990, a extração de madeira e de palmito. Entretanto, os locais selecionados para este estudo não apresentavam vestígios de corte ou queimadas e nem foi observado pés de café no sub-bosque. Assim, as matas selecionadas para este estudo são provavelmente secundárias, mas em bom estado de conservação. 
Amostragem - O total de dez parcelas de $1.200 \mathrm{~m}^{2}$ cada $(30 \times 40 \mathrm{~m})$ foram alocadas aleatoriamente em duas faixas altitudinais, sendo cinco na cota dos 50m.s.m. (sítio 1 ), às margens do rio Penação (c. $20 \mathrm{~m})$, e outras cinco a 250m.s.m. (sítio 2), às margens do rio Opinião (c. $30 \mathrm{~m})$, totalizando 1,2ha amostrados. Em cada parcela foram marcadas e medidas todas as árvores com DAP (diâmetro a altura do peito = $1,30 \mathrm{~m}$ do solo) igual ou superior a $10 \mathrm{~cm}$.

O material botânico coletado foi determinado por comparação com a coleção do herbário do Instituto de Pesquisas Jardim Botânico do Rio de Janeiro (RB), com o auxílio da literatura, e, em alguns casos, enviado para a identificação por especialistas. O material testemunho fértil e/ou uma ou duas exsicatas de cada espécie só com material estéril, foi depositado no herbário da Universidade Estadual do Norte Fluminense, em implantação. Duplicatas foram enviadas aos herbários do Instituto de Pesquisas Jardim Botânico do Rio de Janeiro e da Universidade Federal do Espírito Santo (VIES).

Os parâmetros fitossociológicos estudados foram calculados pelo programa FITOPAC
(Shepherd 1995) e são os usualmente utilizados em trabalhos de fitossociologia, a saber: densidade relativa (DR), dominância relativa (DoR), valor de cobertura (VC), diâmetro médio (DM) e área basal (AB). Os índices de diversidade de espécies de Shannon ( $\mathrm{H}^{\prime}$, calculado utilizando-se o logaritmo neperiano), de equabilidade (J), de similaridade de Jaccard (para comparação com os dados de ocorrência de espécies da literatura) e de Morisita (para comparação entre os sítios estudados) foram calculados segundo Brower \& Zar (1977). Os valores médios de densidade absoluta, área basal por hectare, número de espécies, diversidade ( $\mathrm{H}^{\prime}$ ) e equabilidade (J) entre os sítios amostrados foram comparados estatisticamente pelo Teste t de Student (Zar 1996).

\section{Resultados}

As duas matas estudadas apresentaram valores muito semelhantes, não diferindo estatisticamente ( $p>0,05$, Teste $t)$ em termos de número e diversidade de espécies, número de indivíduos e área basal por hectare (Tab. 1).

Tabela 1. Número de indivíduos (N.Ind), área basal por hectare ( $\mathrm{AB} /$ ha), número de espécies (N.Spp), índice de diversidade de Shannon (H') e índice de equabilidade (J) em cada uma das parcelas amostrais a 50m.s.m. e a 250m.s.m., na região do Imbé, RJ. Não houve diferença estatística entre os valores médios das matas (teste t, $\mathrm{P}<0,05)$ para um mesmo parâmetro.

\begin{tabular}{lccccc}
\hline Parcelas & N.Ind & AB / ha & N.Spp & H' & J \\
\hline P1 & 88 & 49,9 & 48 & 3,56 & 0,92 \\
P2 & 93 & 38,3 & 47 & 3,58 & 0,93 \\
P3 & 88 & 38,5 & 49 & 3,60 & 0,93 \\
P4 & 91 & 41,2 & 42 & 3,22 & 0,86 \\
P5 & 100 & 41,5 & 57 & 3,80 & 0,94 \\
Média \pm DP & $92 \pm 4,9$ & $41.9 \pm 2,5$ & $46,2 \pm 6,1$ & $3,55 \pm 0,21$ & $0,92 \pm 0,03$ \\
Total - 50m.s.m. & 460 & - & 125 & 4,21 & 0,87 \\
P6 & 73 & 28,6 & 38 & 3,42 & 0,94 \\
P7 & 88 & 35,9 & 50 & 3,63 & 0,93 \\
P8 & 89 & 25,8 & 52 & 3,57 & 0,90 \\
P9 & 103 & 43,3 & 57 & 3,82 & 0,95 \\
P10 & 122 & 40,5 & 42 & 3,55 & 0,89 \\
Média \pm DP & $95 \pm 18,4$ & $34,8 \pm 2,2$ & $46,8 \pm 6,6$ & $3,60 \pm 0,15$ & $0,92 \pm 0,02$ \\
Total - 250m.s.m. & 480 & - & 145 & 4,30 & 0,86 \\
\hline
\end{tabular}


Os 940 indivíduos amostrados (460 a 50m.s.m. e 480 a 250m.s.m.) foram distribuídos em 43 famílias (31 e 39, respectivamente), sendo que deste total apenas quatro $(9 \%)$ não foram amostradas a 250m.s.m.: Anacardiaceae, Polygonaceae, Sapindaceae e Thymelaeaceae. Para a mata do sítio 1, este percentual sobe para
$28 \%$ (Tab. 2).

Em relação ao número de espécies por família a 50m.s.m. (Fig. 2, Tab. 2), as mais ricas foram Lauraceae e Leguminosae (17 espécies cada uma), seguidas por Moraceae (11), Myrtaceae (10), Sapotaceae (10), Euphorbiaceae (9) e Rubiaceae (9), enquanto a 250m.s.m., as

Tabela 2. Distribuição das espécies e famílias amostradas em duas zonas altitudinais na região do Imbé, RJ.

\begin{tabular}{|c|c|c|c|c|c|}
\hline Família/Espécie & 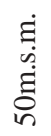 & $\begin{array}{l}\dot{\Xi} \\
\dot{\leftrightarrow} \\
\dot{\Xi} \\
\text { ڤે }\end{array}$ & Família/Espécie & $\begin{array}{l}\dot{\Xi} \\
\dot{\leftrightarrow} \\
\dot{\Xi} \\
\text { م }\end{array}$ & 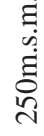 \\
\hline ANACARDIACEAE & & & L. tomentosa (Benth.) Fritsch & $\mathrm{X}$ & \\
\hline Astronium gracile Engl. & $\mathrm{X}$ & & Licania sp. & & $\mathrm{X}$ \\
\hline ANNONACEAE & & & Parinari excelsa Sabine & & $\mathrm{X}$ \\
\hline Annona cacans Warm. & $\mathrm{X}$ & $\mathrm{X}$ & CLUSIACEAE & & \\
\hline Annona sp. & & $\mathrm{X}$ & Tovomitopsis paniculata (Spreng.) Planch. & $X$ & $\mathrm{X}$ \\
\hline Oxandra martiana (Schltdl.) R. E. Fr. & & $\mathrm{X}$ & \& Triana & & \\
\hline O. nitida R. E. Fr. & $\mathrm{X}$ & & COMBRETACEAE & & \\
\hline Rollinia laurifolia Schltdl. & & $\mathrm{X}$ & Terminalia januariensis DC. & & $\mathrm{X}$ \\
\hline Unonopsis sp. & $\mathrm{X}$ & & ELAEOCARPACEAE & & \\
\hline Xylopia laevigata (Mart.) R. E. Fr. & $\mathrm{X}$ & & Sloanea guianensis (Aubl.) Benth. & $\mathrm{X}$ & $\mathrm{X}$ \\
\hline Xylopia sp. & $\mathrm{X}$ & & EUPHORBIACEAE & & \\
\hline APOCYNACEAE & & & Actinostemon verticillatus (K1.) Baill. & $\mathrm{X}$ & $\mathrm{X}$ \\
\hline Geissospermum laeve (Vell.) Miers & $\mathrm{X}$ & $\mathrm{X}$ & Actinostemon sp. & & $\mathrm{X}$ \\
\hline ARALIACEAE & & & Alchornea triplinervia (Spreng.) Müll. Arg. & $\mathrm{X}$ & \\
\hline $\begin{array}{l}\text { Dendropanax arboreum (L.) Decne. } \\
\text { \& Planch. }\end{array}$ & $\mathrm{X}$ & & $\begin{array}{l}\text { Alchornea sp. } 1 \\
\text { Alchornea sp. } 2\end{array}$ & $\mathrm{X}$ & $\mathrm{X}$ \\
\hline Didymopanax morototoni (Aubl.) Decne. & & $\mathrm{X}$ & Aparisthmium cordatum (Juss.) Baill. & $\mathrm{X}$ & $\mathrm{X}$ \\
\hline \& Planch. & & & Hyeronima alchorneoides Allemão & $\mathrm{X}$ & $\mathrm{X}$ \\
\hline BIGNONIACEAE & & & Mabea fistulifera Mart. & $\mathrm{X}$ & $\mathrm{X}$ \\
\hline Tabebuia obtusifolia (Charm.) Bureau & & $\mathrm{X}$ & Pausandra morisiana (Casar.) Radlk. & & $\mathrm{X}$ \\
\hline BOMBACACEAE & & & Pausandra sp. & $\mathrm{X}$ & \\
\hline $\begin{array}{l}\text { Eriotheca pentaphylla (Vell. emend. K. } \\
\text { Schum.) A. Robyns }\end{array}$ & $\mathrm{X}$ & $\mathrm{X}$ & $\begin{array}{l}\text { Pera glabrata (Schott) Baill. } \\
\text { Phyllanthus umbratus Müll. Arg. }\end{array}$ & $\mathrm{X}$ & $\mathrm{X}$ \\
\hline E. candolleana (K. Schum.) A. Robyns & & $\mathrm{X}$ & Senefeldera multiflora Mart. & $\mathrm{X}$ & \\
\hline BURSERACEAE & & & FLACOURTIACEAE & & \\
\hline Protium widgrenii Engl. & $\mathrm{X}$ & $\mathrm{X}$ & Casearia arborea (L. C. Rich.) Urb. & $\mathrm{X}$ & \\
\hline CARICACEAE & & & Casearia sp. & & $\mathrm{X}$ \\
\hline Jacaratia heptaphylla (Vell.) A. DC. & & $\mathrm{X}$ & ICACINACEAE & & \\
\hline J. spinosa (Aubl.) A. DC. & $\mathrm{X}$ & & Citronella paniculata (Mart.) R. A. Howard & & $\mathrm{X}$ \\
\hline CELASTRACEAE & & & LAURACEAE & & \\
\hline Maytenus commutata Reiss. & & $\mathrm{X}$ & Aniba firmula (Nees \& Mart.) Mez & $\mathrm{X}$ & $\mathrm{X}$ \\
\hline M. communis Reiss. & & $\mathrm{X}$ & Beilschmiedia emarginata (Meisn.) & $\mathrm{X}$ & \\
\hline CHRYSOBALANACEAE & & & Kosterm. & & \\
\hline Hirtella hebeclada Moric. ex A. DC. & $\mathrm{X}$ & & Beilschmiedia sp. & $\mathrm{X}$ & \\
\hline Licania kunthiana Hook. f. & & $\mathrm{X}$ & Cryptocarya micrantha Meisn. & $\mathrm{X}$ & \\
\hline
\end{tabular}


Tabela 2 (continuação)

Família/Espécie

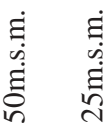

LAURACEAE

C. minima $\mathrm{Mez}$

C. moschata Nees \& Mart. ex Nees

Cryptocarpa saligna $\mathrm{Mez}$

Licaria armeniaca (Nees) Kosterm.

Nectandra grandiflora Nees

N. leucotirsus Meisn.

Nectandra sp.

Ocotea aciphylla (Nees) Mez

O. aniboides $\mathrm{Mez}$

O. daphnifolia (Meisn.) Mez

O. diospyrifolia (Meisn.) Mez

O. dispersa (Nees) Mez

O. divaricata (Nees) Mez

O. insignis $\mathrm{Mez}$

O. martiana $\mathrm{Mez}$

O. odorifera (Vell.) Rohwer

Ocotea sp. 1

Ocotea sp. 2

Phyllostemonodaphne geminiflora (Meisn.)

Kosterm.

Lauraceae sp. 1

Lauraceae sp. 2

LECYTHIDACEAE

Cariniana estrelensis (Raddi) Kuntze

Couratari pyramidata (Vell.) Knuth

Lecythis lanceolata Poir.

\section{LEGUMINOSAE}

\section{MIMOSOIDEAE}

Abarema sp. nova

Abarema sp.

Inga dulcis (Vell.) Mart.

I. thibaudiana DC.

I. organensis Pittier

Inga sp.

Plathymenia foliolosa Benth.

Pseudopiptadenia contorta (DC.) G. P. $\quad$ X

Lewis \& M. P. Lima

Leguminosae sp. 1

CAESALPINIOIDEAE

Chamaecrista ensiformis (Vell.) Irwin

\& Barneby

Copaifera langsdorfii Desf.

Hymenaea sp.

Melanoxylon brauna Schott

Peltogyne mattosiana Rizzini

Sclerolobium striatum Dwyer

Sclerolobium sp.

Tachigali paratyensis (Vell.) H. C. Lima

$\mathrm{X}$
$\mathrm{X}$

$\mathrm{X}$

$\mathrm{X}$

$\mathrm{X}$

$\mathrm{X}$

$\mathrm{X}$

$\mathrm{X}$

$\mathrm{X}$

$\begin{array}{ll}X & X \\ X & \end{array}$

X $\quad X$

Família/Espécie

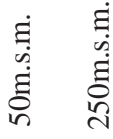

\section{FABOIDEAE}

Andira fraxinifolia Benth.

Luetzelburgia trialata Ducke

Machaerium sp.

Myrocarpus frondosus Allemão

Pterocarpus rohrii Vahl

Ormosia sp.

Swartzia flaemingii Raddi

Vataireopsis araroba (Aguiar) Ducke

Zollernia splendens Nees

MELASTOMATACEAE

Miconia prasina (Sw.) DC.

MELIACEAE

Cabralea canjerana (Vell.) Mart.

Guarea kunthiana A. Juss.

Trichilia elegans A. Juss.

X

$\mathrm{X}$

$\mathrm{X}$

$\mathrm{X}$

$\mathrm{X}$

$\mathrm{X}$

$\mathrm{X}$

$\mathrm{X}$

T. lepidota Mart.

T. martiana C. DC

T. silvatica C. DC.

Trichilia sp.

MONIMIACEAE

Mollinedia cyathantha Perkins

$M$. heteranthera Perkins

M. lamprophylla Perkins

M. oligantha Perkins

M. pachysandra Perkins

M. puberula Perkins

Mollinedia sp.

Siparuna reginae (Tul.) A. DC.

Siparuna sp.

MORACEAE

Brosimum glaziovii Taub.

B. guianense (Aubl.) Huber

Cecropia pachystachya Trécul

Coussapoa curranii Blake

Ficus citrifolia P. Miller

F. gomelleira Kunth \& Bouché ex Kunth

F. pulchella Schott

Ficus sp. 1

Ficus sp. 2

Ficus sp. 3

$\mathrm{X}$

$\mathrm{X}$

X

$\mathrm{X}$

$\mathrm{X}$

$\mathrm{X}$

X

$\mathrm{X}$

$\mathrm{X}$

X X

X

$\mathrm{X}$

$\mathrm{X}$

$\mathrm{X}$

$\mathrm{X}$

X

$\mathrm{X}$

X $\quad X$

$\mathrm{X}$

X

X $\quad X$

X $\quad X$

$\mathrm{X}$

$\mathrm{X}$

$\mathrm{X}$

X X

$\mathrm{X}$

$\mathrm{X}$

$\mathrm{X}$

$\mathrm{X}$

$\begin{array}{lll}\mathrm{X} & \mathrm{X}\end{array}$ Rusby

Naucleopsis mello-barretoi (Standl.) $\quad$ X $\quad$ X

C. C. Berg

Pseudolmedia hirtula Kuhlm.

Pourouma guianensis Aubl.

Sorocea guilleminiana Gaudich.

Sorocea sp.

$\begin{array}{ll}X & X \\ X & x\end{array}$

$\begin{array}{ll}X & X \\ X & x\end{array}$

X X 
Tabela 2 (continuação)

Família/Espécie

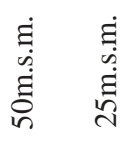

Família/Espécie

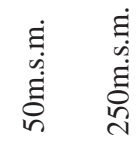

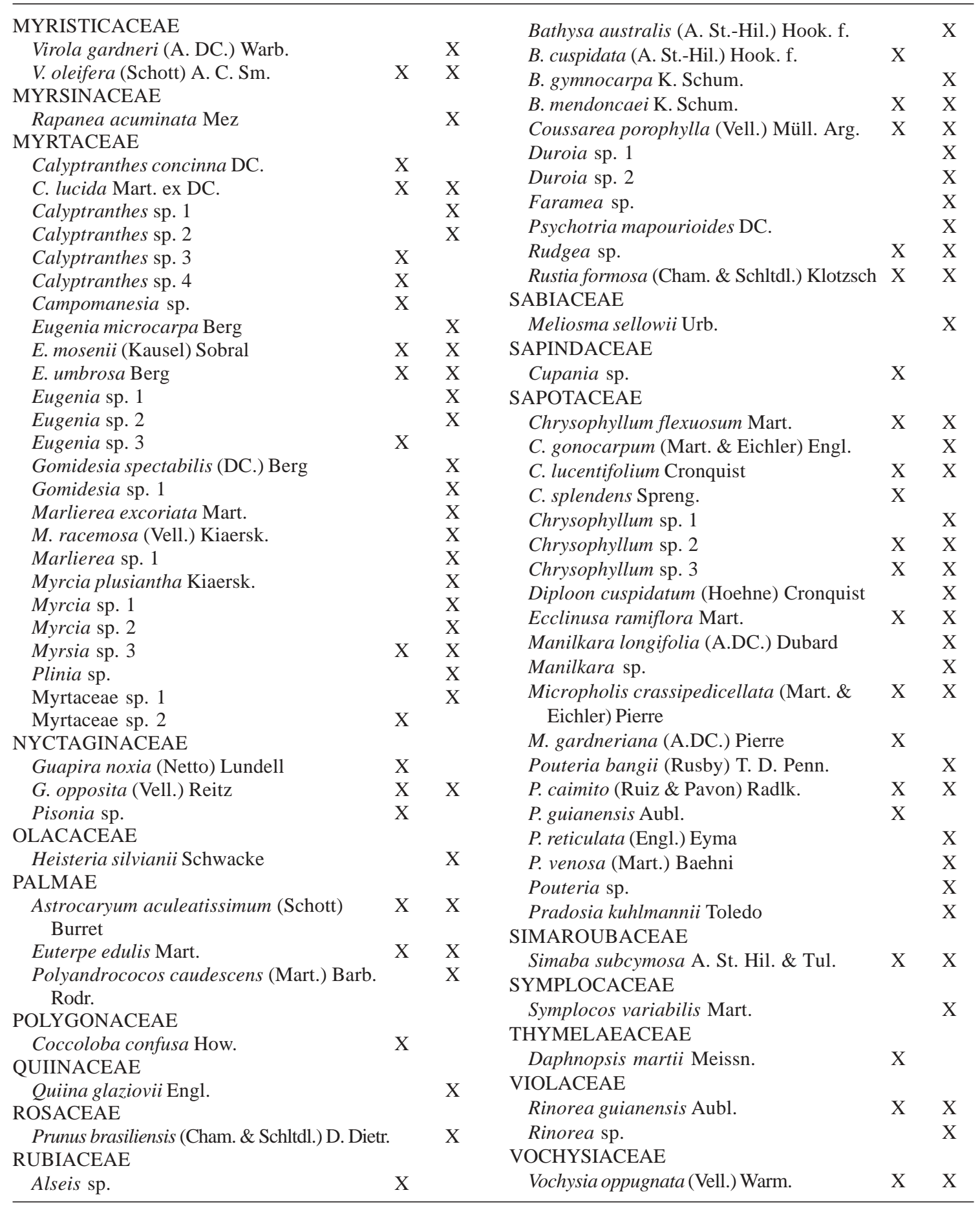


mais ricas foram Myrtaceae (19), Leguminosae (17), Sapotaceae (17), Lauraceae (16) e Moraceae (13).

Euphorbiaceae apresentou o maior VC nos dois sítios amostrados, devido ao grande número de indivíduos. Entretanto, esta família não apresentou a maior riqueza de espécies em nenhuma das áreas, sendo, a 50m.s.m., superada por Lauraceae, Leguminosae, Moraceae, Myrtaceae e Sapotaceae e por Myrtaceae, Leguminosae, Sapotaceae, Lauraceae e Moraceae, na mata a 250m.s.m. (Fig. 2, Tab. 2). Com relação ao VC, Euphorbiaceae foi seguida, respectivamente, por Leguminosae, Rubiaceae, Moraceae e Sapotaceae, na mata a $50 \mathrm{~m} . \mathrm{s} . \mathrm{m}$. , e por Leguminosae, Palmae, Sapotaceae e Lauraceae, a 250m.s.m. (Fig. 3).

Dentre as famílias de maior destaque, a que apresentou maiores diferenças na representatividade entre os dois sítios foi Rubiaceae, que ocorre principalmente no sub-bosque. As duas espécies mais representativas na amostragem geral, Rustia formosa e Bathysa mendoncaei, ocorreram de forma muito mais significativa na mata a 50m.s.m. (Tab. 3 e 4).

Os dois sítios diferiram em termos de composição específica, com apenas 57 espécies comuns das 210 coletadas (Tab. 3 e 4). O dendrograma de similaridade de espécies entre as parcelas amostrais (Fig. 4) demonstrou claramente a existência de dois grupos: 1) parcelas a $50 \mathrm{~m} . \mathrm{s} . \mathrm{m}$. e 2) parcelas a $250 \mathrm{~m} . \mathrm{s} . \mathrm{m}$., com uma maior semelhança entre as parcelas da mata a $50 \mathrm{~m} . \mathrm{s} . \mathrm{m}$. O valor do índice de similaridade de Morisita entre os dois sítios foi de 0,46 .

Dentre as 15 espécies de maior cobertura na amostragem geral, algumas foram bem representadas nos dois sítios, como é o caso de Actimostemon verticilatus, Mabea fistulifera e Astrocaryum aculeatissimum (Tab. 3 e 4). Outras foram melhor representadas na mata a 50m.s.m., como Hyeronima alchorneoides, Rustia formosa, Virola oleifera, Pseudopiptadenia contorta e Bathysa mendoncaei, ou na mata a 250m.s.m., como Euterpe edulis, Ecclinusa ramiflora, Vochysia oppugnata, Aniba firmula e Maytenus commutata, (Tab. 3 e 4).

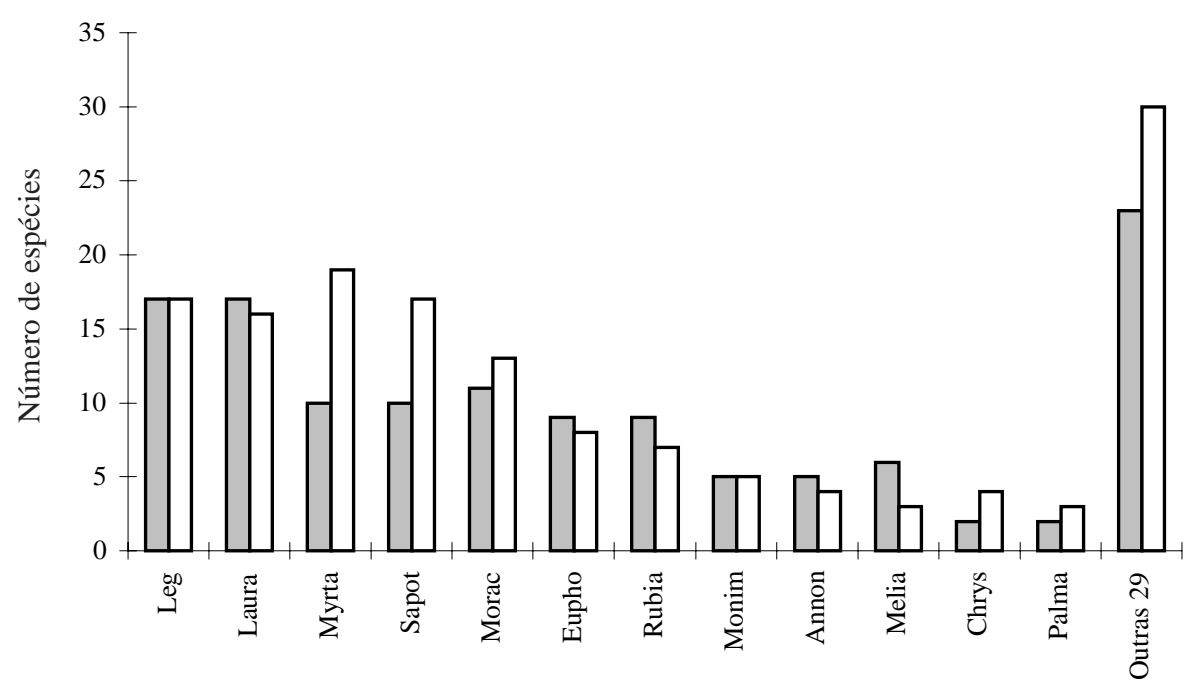

Figura 2. Distribuição das doze famílias com maior número de espécies nos dois sítios estudados (a 50 e $250 \mathrm{~m}$ de altitude), na região do Imbé, RJ (Leg = Leguminosae; Laura = Lauraceae; Myrta = Myrtaceae; Sapot = Sapotaceae; Morac $=$ Moraceae $;$ Eupho $=$ Euphorbiaceae $;$ Rubia $=$ Rubiaceae $;$ Monim = Monimiaceae $;$ Annon = Annonaceae $;$ Melia $=$ Meliaceae; Chrys $=$ Chrysobalanaceae; Palma $=$ Palmae $) . \quad=50 \mathrm{~m} ; \square=250 \mathrm{~m}$. 


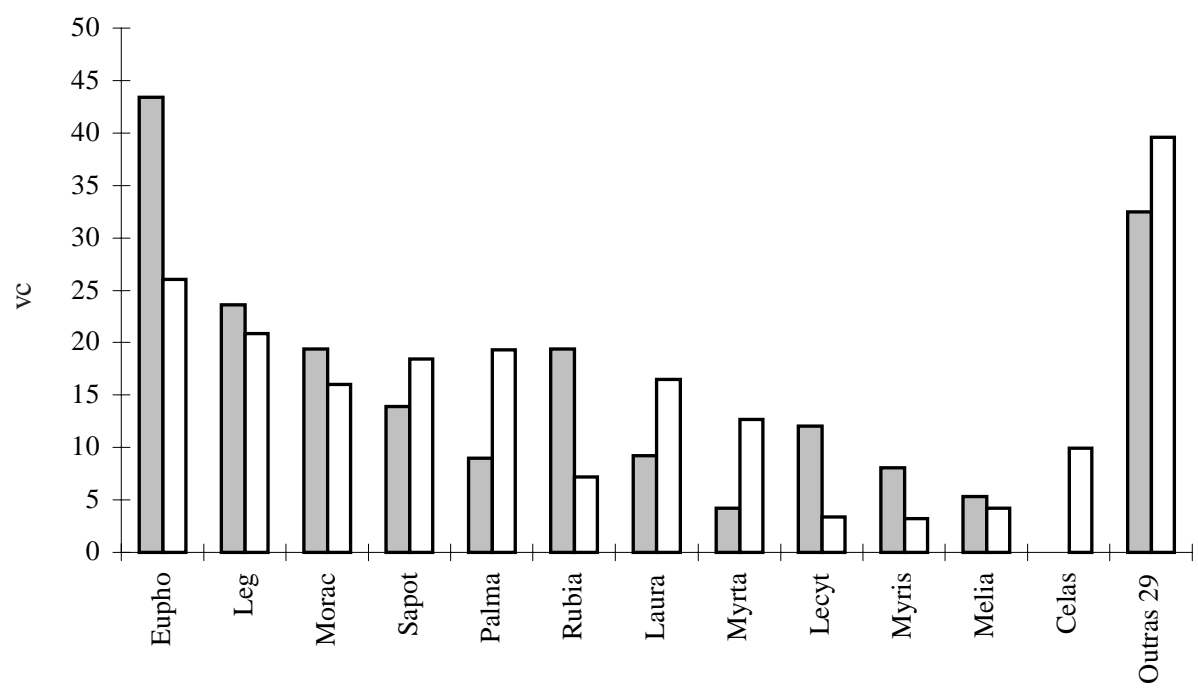

Figura 3. Distribuição das doze famílias de maior VC nos dois sítios estudados (a 50 e 250m.s.m. de altitude), na região do Imbé, RJ (Eupho = Euphorbiaceae; Leg = Leguminosae; Morac = Moraceae; Sapot = Sapotaceae $;$ Rubia $=$ Rubiaceae; Lecyt $=$ Lecythidaceae; Laura $=$ Lauraceae $;$ Myris = Myristicaceae $;$ Celas = Celastraceae Myrta = Myrtaceae; Palma $=$ Palmae; Melia $=$ Meliaceae). $\square=50 \mathrm{~m} ; \square=250 \mathrm{~m}$.

Entre as 15 principais espécies em $\mathrm{VC}$ nas duas matas, nota-se que apenas cinco, Actimostemon verticilatus, Mabea fistulifera, Euterpe edulis, Ecclinusa ramiflora e Astrocaryum aculeatissimum, foram comuns a ambas. As outras espécies diferiram em densidade e dominância relativa entre os sítios. Este fato ressalta ainda mais as diferenças encontradas entre as zonas altitudinais. Espécies com destaque em um sítio foram pouco representativas no outro, como ocorreu com Hyeronima alchorneoides, abundante a 50m.s.m. (sítio 1), ou até mesmo ausentes, como foi o caso de Polyandrococos caudescens, que só ocorreu na mata a 250m.s.m. (sítio 2).

\section{Discussão}

Os valores de 41,9m² (sítio 1) e $34,8 \mathrm{~m}^{2}$ (sítio 2) de área basal por hectare (árvores com DAP $\geq 10 \mathrm{~cm}$ ) estão entre os mais altos quando comparados com dados de outras florestas (Tab. 5), podendo ser considerado indicativo da preservação da área.

Os dados estruturais (ex: área basal $>30 \mathrm{~m}^{2} \cdot \mathrm{ha}^{-1}$ ), o baixo número de árvores mortas em pé e a ausência de vestígios de árvores cortadas dentro das parcelas, aliados a presença de epífitas em árvores de grande porte, principalmente na mata do sítio 2 , indicam que as matas estudadas, embora secundárias, podem ser consideradas maduras, segundo critérios de Clark (1996).

Os índices de Shannon $\left(\mathrm{H}^{\prime}\right)$ para as áreas estudadas (4,21 a 50m.s.m. e 4,30 a 250m.s.m.) encontram-se entre os maiores valores obtidos para Mata Atlântica, utilizando-se o limite mínimo de inclusão igual a $10 \mathrm{~cm}$ de DAP (Martins 1993). Os dados sugerem que as matas estudadas no Imbé apresentam diversidade local maior que a encontrada para as matas da Serra de Macaé de Cima - área de mata preservada $\left(\mathrm{H}^{\prime}=4,05\right)$ (Guedes-Bruni et al. 1997) ou secundária $\left(\mathrm{H}^{\prime}=3,66\right)$ (Pessoa et al. 1997) - e da mata da Estação Ecológica do Paraíso (H' = 4,20) (Kurtz \& Araújo 2000), visto que, para estas matas, utilizou-se o critério mínimo de inclusão de $5 \mathrm{~cm}$ de DAP. As matas do Imbé também apresentaram maior diversidade de espécies quando comparadas com a floresta estacional semidecidual do Carvão ( $\mathrm{H}^{\prime}=3,21$ ), área de tabuleiros terciários (Silva \& Nascimento 
Tabela 3. Parâmetros fitossociológicos para as espécies amostradas em 0,6 ha na mata a 50m.s.m. na região do Imbé, RJ, ordenadas pelo VC $\left(\mathrm{N}\right.$. ind = número de indivíduos; Ar. Bas. = área basal $\left(\mathrm{m}^{2}\right)$; Dens. $\mathrm{Re}=$ densidade relativa; Dom. Rel $=$ dominância relativa; $\mathrm{VC}=$ valor de cobertura).

\begin{tabular}{|c|c|c|c|c|c|}
\hline Espécie & N. Ind & Ar. Bas. & Dens. $R e$ & Dom. Rel & $\mathrm{VC}$ \\
\hline Hyeronima alchorneoides & 19 & 2,56 & 4,1 & 10,2 & 14,3 \\
\hline Actimostemon verticilatus & 39 & 0,72 & 8,5 & 2,9 & 11,4 \\
\hline Rustia formosa & 26 & 1,26 & 5,7 & 5,0 & 10,7 \\
\hline Virola oleifera & 14 & 1,28 & 3,0 & 5,1 & 8,1 \\
\hline Mabea fistulifera & 17 & 0,94 & 3,7 & 3,8 & 7,5 \\
\hline Pseudopiptadenia contorta & 9 & 1,29 & 2,0 & 5,2 & 7,1 \\
\hline Euterpe edulis & 21 & 0,22 & 4,6 & 0,9 & 5,4 \\
\hline Bathysa mendoncaei & 16 & 0,47 & 3,5 & 1,9 & 5,3 \\
\hline Plathymenia foliosa & 4 & 1,05 & 0,9 & 4,2 & 5,1 \\
\hline Lecythis lanceolata & 6 & 0,87 & 1,3 & 3,5 & 4,8 \\
\hline Couratari pyramidata & 4 & 0,94 & 0,9 & 3,8 & 4,6 \\
\hline Ecclinusa ramiflora & 11 & 0,36 & 2,4 & 1,5 & 3,8 \\
\hline Helicostylis tomentosa & 10 & 0,38 & 2,2 & 1,5 & 3,7 \\
\hline Alchornea sp. 1 & 5 & 0,64 & 1,1 & 2,5 & 3,6 \\
\hline Astrocaryum aculeatissimum & 13 & 0,18 & 2,8 & 0,7 & 3,5 \\
\hline Chrysophyllum sp. 3 & 7 & 0,47 & 1,5 & 1,9 & 3,4 \\
\hline Ficus sp. 3 & 3 & 0,69 & 0,7 & 2,7 & 3,4 \\
\hline Pausandra sp. & 10 & 0,26 & 2,2 & 1,0 & 3,2 \\
\hline Sorocea guillerminiana & 8 & 0,34 & 1,7 & 1,3 & 3,1 \\
\hline Rinoria guianensis & 6 & 0,36 & 1,3 & 1,4 & 2,7 \\
\hline Chrysophyllum flexuosum & 6 & 0,36 & 1,3 & 1,4 & 2,7 \\
\hline Cabralea cangerana & 6 & 0,36 & 1,3 & 1,4 & 2,7 \\
\hline Brosimum glasiovii & 7 & 0,29 & 1,5 & 1,2 & 2,7 \\
\hline Cariniana estrelensis & 5 & 0,38 & 1,1 & 1,5 & 2,6 \\
\hline Ficus gomeleira & 3 & 0,35 & 0,7 & 1,4 & 2,0 \\
\hline Outras 100 espécies & 185 & 8,12 & 40,0 & 32,1 & 72,6 \\
\hline Total & 460 & 25,14 & 100,0 & 100,0 & 200,0 \\
\hline
\end{tabular}

2001), formação esta que tem sido considerada bastante diferenciada das áreas de Mata Atlântica de encosta (Soares \& Ascoly 1970; Silva \& Nascimento 2001). Os valores do Imbé foram superados apenas pelos obtidos em Santa Lúcia $\left(H^{\prime}=5,11\right)$, reserva florestal na região central do Espírito Santo (Thomas 1998), onde se utilizou o critério mínimo de inclusão de $5 \mathrm{~cm}$ de DAP. Este resultado corrobora a idéia de que a Mata Atlântica apresenta grande riqueza florística, heterogeneidade e diversidade (Martins 1989), cujos índices observados estão entre os maiores para o território nacional (Tab. 5).

Algumas famílias, como Euphorbiaceae, Rubiaceae e Palmae possuem altos VCs relacionados principalmente a um pequeno grupo de espécies (Euphorbiaceae $=A$. ver ticilatus, $H$. alchorneoides e $M$. fistulifera, Rubiaceae $=R$. formosa e Bathysa mendoncaei e Palmae ocorrendo com apenas 3 espécies, sendo a principal $E$. edulis), o que faz com que exista certa dominância ao nível de família, característica que parece ser comum em florestas tropicais (Richards 1981). No entanto, outras famílias como Leguminosae e Lauraceae não apresentam esta característica tão clara, primando principalmente por sua diversidade de espécies relativamente alta.

O domínio das Euphorbiaceae nos dois sítios amostrados (50m.s.m. e 250m.s.m.) é também 
Tabela 4. Parâmetros fitossociológicos para as espécies amostradas em 0,6 ha na mata a 250m.s.m. na Região do Imbé, $\mathrm{RJ}$, ordenadas pelo VC (N. ind= número de indivíduos; Ar. Bas.= área basal; Dens. Re= densidade relativa; Dom. Rel = dominância relativa; $\mathrm{VC}=$ valor de cobertura).

\begin{tabular}{|c|c|c|c|c|c|}
\hline Espécie & N. Ind & Ar. Bas. & Dens. Re & Dom. Rel & $\mathrm{VC}$ \\
\hline Actimostemon verticilatus & 45 & 0,92 & 9,4 & 4,4 & 13,8 \\
\hline Euterpe edulis & 47 & 0,53 & 9,8 & 2,6 & 12,4 \\
\hline Mabea fistulifera & 17 & 0,96 & 3,5 & 4,6 & 8,1 \\
\hline Ecclinusa ramiflora & 18 & 0,72 & 3,8 & 3,4 & 7,2 \\
\hline Vochysia oppugnata & 9 & 0,88 & 1,9 & 4,2 & 6,1 \\
\hline Maytenus commutata & 7 & 0,94 & 1,5 & 4,5 & 6,0 \\
\hline Aniba firmula & 14 & 0,37 & 2,9 & 1,8 & 4,7 \\
\hline Sclerolobium striatum & 3 & 0,80 & 0,6 & 3,8 & 4,5 \\
\hline Polyandrococos caudescens & 15 & 0,19 & 3,1 & 0,9 & 4,0 \\
\hline Maytenus communis & 6 & 0,55 & 1,3 & 2,7 & 3,9 \\
\hline Calyptrantes lucida & 9 & 0,38 & 1,9 & 1,8 & 3,7 \\
\hline Pouroma guianensis & 8 & 0,43 & 1,7 & 2,0 & 3,7 \\
\hline Cariniana estrelensis & 2 & 0,62 & 0,4 & 3,0 & 3,4 \\
\hline Astrocaryum aculeatissimum & 10 & 0,18 & 2,1 & 0,9 & 3,0 \\
\hline Lauraceae sp. 1 & 6 & 0,35 & 1,3 & 1,7 & 2,9 \\
\hline Licania kunthiana & 5 & 0,39 & 1,0 & 1,9 & 2,9 \\
\hline Virola oleifera & 7 & 0,23 & 1,5 & 1,1 & 2,6 \\
\hline Peltogyne mattosiana & 5 & 0,31 & 1,0 & 1,5 & 2,5 \\
\hline Helicostylis tomentosa & 6 & 0,27 & 1,3 & 1,3 & 2,5 \\
\hline Cabralea cangerana & 5 & 0,30 & 1,0 & 1,4 & 2,5 \\
\hline Simaba subcimosa & 5 & 0,28 & 1,0 & 1,4 & 2,4 \\
\hline Rustia formosa & 4 & 0,29 & 0,8 & 1,4 & 2,2 \\
\hline Geissospermum laevis & 2 & 0,34 & 0,4 & 1,6 & 2,1 \\
\hline Chrysophyllum flexuosum & 5 & 0,21 & 1,0 & 1,0 & 2,0 \\
\hline Pausandra morisiana & 7 & 0,12 & 1,5 & 0,6 & 2,0 \\
\hline Outras 120 espécies & 213 & 9,32 & 44,3 & 44,5 & 88,9 \\
\hline Total & 480 & 20,88 & 100,0 & 100,0 & 200,0 \\
\hline
\end{tabular}

demonstrado em alguns trabalhos já realizados em Mata Atlântica (Silva 1980; Jarenkow 1994).

Myrtaceae vem sendo destacada em diversos trabalhos (Mori et al. 1983; Leitão-Filho 1987; Grombone et al. 1990; Melo 1993; Mantovani 1993; Melo \& Mantovani 1994; Jarenkow 1994; Melo et al. 1998; Kurtz \& Araújo 2000) como família que assume importância muito grande nos complexos florestais próximos à costa brasileira. Normalmente, na Mata Atlântica esta família encontra-se listada entre as de maior riqueza (Peixoto 1992), fato observado no presente estudo.

Os gêneros com maior número de espécies amostrados neste trabalho, Ocotea, Mollinedia,
Chrysophyllum, Ficus, Calyptranthes, Eugenia, Pouteria e Trichilia, estão diferentemente representados em outros trabalhos de fitossociologia e florística realizados em Mata Atlântica no Estado do Rio de Janeiro (Pessoa et al. 1997; Guedes-Bruni et al. 1997; Kurtz \& Araújo 2000), sugerindo ausência de padrão de distribuição destes gêneros no Estado do Rio de Janeiro.

Nos dois sítios ocorreu maior cobertura de algumas espécies, normalmente formando um grupo com VCs superiores a 10, com pequenas diferenças entre si, mas relativamente distantes das demais espécies. Este destaque em cobertura, no entanto, é muito pequeno quando comparado com diferentes ecossistemas 
Nível de fusão

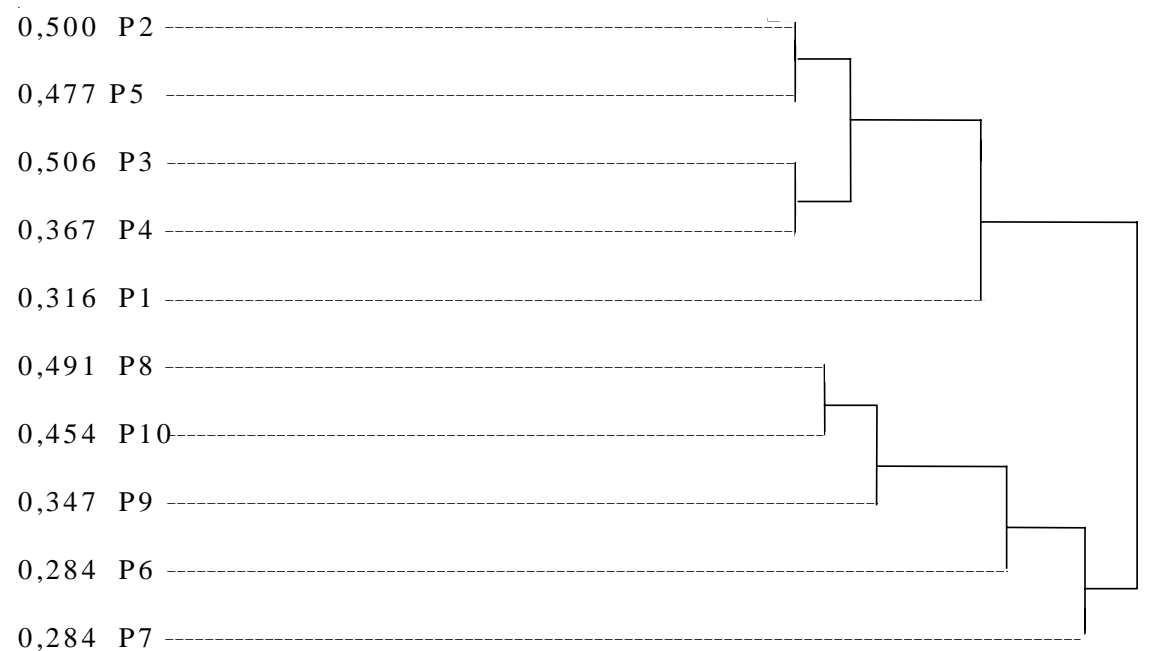

Figura 4. Dendrograma de similaridade de espécies para as parcelas (P1 a P5) do sítio 1 (50m.s.m.) e as parcelas (P6 a P7) do sítio 2 (250m.s.m.), na região do Imbé, RJ, a partir do coeficiente de Morisita (mod. horn). Método de agrupamento: Média de grupo (UPGMA).

brasileiros, que podem apresentar áreas onde o predomínio de uma ou de poucas espécies é bem mais pronunciado (Nascimento \& Cunha 1989, Pantanal; Nascimento \& Saddi 1992, Cerrado; Nascimento et al. 1997, Mata Amazônica de Terra Firme).

A similaridade de espécies entre as zonas altitudinais estudadas não é grande, com apenas 57 espécies comuns, o que corresponde a similaridade de 46\% (índice de Morisita). As principais espécies estão representadas nos dois sítios, o que poderia passar a idéia de semelhança entre eles. No entanto, observa-se que, na maioria dos casos, o VC destas espécies é diferente, sendo mais representativas em um dos sítios. De forma geral, as duas matas apresentam diferenças na composição bastante significativas, principalmente no aspecto qualitativo.

Existem muitas formas pelas quais as espécies podem ser consideradas raras, padrão este com profundas consequiências ecológicas e evolutivas (Rabinowitz 1981). Tomando-se como base para a determinação de espécies raras aquelas que são representadas por apenas um indivíduo (Martins 1993) na área de estudo,
60 espécies (48\%) na mata a 50m.s.m. e 73 espécies (50\%) na mata a $250 \mathrm{~m} . s . m$. encontravam-se nesta categoria, o que é um número bastante significativo e esperado para sistemas tão diversos quanto os de Mata Atlântica, onde se observam também elevados índices de endemismo (Mori et al. 1981). Deve-se considerar, no entanto, que a classificação a partir do número de espécies amostradas pode ser muito relativa, uma vez que determinada espécie com abundância relativamente alta na área de estudo pode apresentar distribuição geográfica muito restrita ou, por outro lado, uma espécie pouco representada na área ter ampla distribuição. De qualquer forma, alguns estudos têm demonstrado que espécies que apresentam ampla distribuição comumente possuem maiores abundâncias locais que espécies que possuem distribuição geográfica mais restrita (Gaston \& Lawton 1990).

Das 15 espécies com maiores VCs na amostragem geral, Hyeronima alchorneoides, Rustia formosa, Virola oleifera, Astrocaryum aculeatissimum, Euterpe edulis e Ecclinusa ramiflora foram amostradas na mata da 
Tabela 5. Dados estruturais obtidos em alguns trabalhos realizados em Mata Atlântica (Área = área amostral total em ha; DAP = diâmetro a altura do peito da amostragem; H' = índice de diversidade de Shannon; N. spp. = número de espécies; den. $=$ densidade por hectare; $\mathrm{AB}=$ área basal por hectare $-\mathrm{m}^{2} \mathrm{ha}^{-1}$ ). Matas preservadas $=1$; matas impactadas $=2$.

\begin{tabular}{|c|c|c|c|c|c|c|c|}
\hline Local & Fonte & Área & DAP & $\mathrm{H}^{\prime}$ & N.spp & den & $\mathrm{AB}$ \\
\hline Ubatuba - SP (área I) ${ }^{1}$ & Silva 1980 & 1,0 & $\geq 10$ & 3,49 & 64 & 819 & - \\
\hline Ubatuba - SP (área II $)^{1}$ & Silva 1980 & 1,0 & $\geq 10$ & 4,03 & 94 & 814 & - \\
\hline Ubatuba - SP $(\text { geral })^{1}$ & Silva 1980 & 2,0 & $\geq 10$ & 4,07 & 123 & 816 & - \\
\hline Juréia - SP ${ }^{1}$ & Mantovani 1993 & 1,0 & $\geq 9,5$ & 4,19 & 155 & 882 & - \\
\hline Paraíso - RJ ${ }^{1}$ & Kurtz \& Araújo 2000 & - & $\geq 5$ & 4,20 & 138 & 1.370 & 57,2 \\
\hline Morrinhos do Sul - RS ${ }^{1}$ & Jarenkow 1994 & 1,0 & $\geq 10$ & 3,88 & 97 & 1.127 & 42,6 \\
\hline Vale do Sol - RS ${ }^{1}$ & Jarenkow 1994 & 1,0 & $\geq 10$ & 2,63 & 52 & 782 & 37,2 \\
\hline Macaé de Cima - RJ ${ }^{1}$ & Guedes-Bruni et al. 1997 & 1,0 & $\geq 5$ & 4,05 & 189 & 2.288 & - \\
\hline Macaé de Cima - RJ² & Pessoa et al. 1997 & 1,0 & $\geq 5$ & 3,66 & 157 & 2.217 & 27,9 \\
\hline Mergulhão - RJ² & Nascimento, não public. & 0,6 & $\geq 10$ & 3,81 & 69 & 465 & 18,2 \\
\hline Santa Lúcia-ES ${ }^{1}$ & Thomas 1998 & 0,3 & $\geq 5$ & 5,11 & 228 & 1.995 & - \\
\hline Mata do Carvão - $\mathrm{RJ}^{1}$ & Silva \& Nascimento 2001 & 1,0 & $\geq 10$ & 3,21 & 84 & 564 & 15,0 \\
\hline Juréia-Itatins - SP${ }^{1}$ & Melo et al. 1998 & 1,0 & $\geq 5$ & 4,21 & 173 & - & - \\
\hline Imbé - RJ (50m.s.m.) ${ }^{1}$ & Presente estudo & 0,6 & $\geq 10$ & 4,21 & 125 & 767 & 41,9 \\
\hline Imbé - RJ (250m.s.m. $)^{1}$ & Presente estudo & 0,6 & $\geq 10$ & 4,30 & 145 & 792 & 34,6 \\
\hline
\end{tabular}

Estação Ecológica do Paraíso, sendo que E. edulis é a mais abundante e a segunda em termos de VI (Kurtz \& Araújo 2000). Já a mata de Macaé de Cima (Guedes-Bruni et al. 1997) apresentou apenas uma espécie em comum, Euterpe edulis. Para as espécies da mata do Carvão, somente Pseudopiptadenia contorta, segunda em termos de densidade, aparece entre as amostradas na mata do Imbé.

Os resultados demonstram que as matas do Imbé apresentam maior similaridade florística (coeficiente de Jaccard $\left(S_{\mathrm{j}}\right)=0,21$ e 0,19$)$ (Fig. 5) com a mata da Estação Ecológica Estadual do Paraíso, que, embora ocorrendo a maior distância, está em altitude semelhante ao complexo do Imbé, com 200m.s.m., contra aproximadamente $1.100 \mathrm{~m}$ de Macaé de Cima e $10 \mathrm{~m}$ da mata do Carvão. Estes valores superam mais do que em dobro todos os valores encontrados para todas as outras comparações (Fig. 5) e se aproximam mais da similaridade encontrada para as duas matas estudadas no Imbé $\left(\mathrm{S}_{\mathrm{j}}=0,31\right)$. Assim, a maior similaridade entre as matas do Imbé e a mata da Estação
Ecológica do Paraíso deve estar diretamente relacionada com a altitude. A baixa similaridade encontrada com a mata do Carvão, a mais próxima geograficamente da região de estudo, está muito provavelmente relacionada ao fato desta última ser outro tipo de formação vegetal e, assim, possuir flora bastante distinta.

Os resultados encontrados indicam que as duas zonas altitudinais estudadas apresentam semelhanças estruturais (ex. densidade arbórea e área basal), mas diferem quanto à composição florística.

Euterpe edulis é espécie classificada como clímax (Mantovani 1993), podendo ser considerada bom indicativo de preservação de matas. Assim, grande número de indivíduos de E. edulis amostrados e a não ocorrência de vestígios de corte ou fogo, além de altos valores de área basal nas duas matas, indicam que os dois sítios estudados estão em bom estado de conservação e podem ser considerados representativos da composição arbórea que, no passado, recobria toda a região da Serra do Imbé. 


\begin{tabular}{l|ccccc} 
Imbé (250m.s.m.) & - & & & & \\
Imbé (50m.s.m.) & 0,31 & - & & & \\
Paraíso & 0,21 & 0,19 & - & & \\
Macaé de Cima & 0,07 & 0,05 & 0,06 & - & \\
Carvão & 0,05 & 0,04 & 0,04 & 0,02 & - \\
\cline { 2 - 6 } & Imbé & Imbé & Paraíso & Macaé & Carvão \\
& $(250 m . s . m)$. & (50m.s.m.) & & de Cima &
\end{tabular}

Figura 5. Coeficientes de similaridade de Jaccard ( $\mathrm{S}_{\mathrm{j}}$ ) comparando os dois sítios estudados (a 50 e $250 \mathrm{~m}$ de altitude) na região do Imbé, RJ e outras regiões de Mata Atlântica de encosta e mata de tabuleiro no estado do Rio de Janeiro, considerando-se apenas as espécies identificadas ao nível específico.

\section{Agradecimentos}

À FENORTE, pela concessão da bolsa de Mestrado; ao CNPq e a FINEP, pelo financiamento do projeto; ao Sr. Armando Chaves, proprietário da Fazenda Opinião, onde a área de estudo está localizada; aos profissionais do Instituto de Pesquisas Jardim Botânico do Rio de Janeiro, Haroldo C. de Lima, Marli P. Morim de Lima, Angela M. S. da Fonseca Vaz, José Fernando A. Baumgratz, Nilda M. F. da Silva, Marcus N. Coelho, Alexandre Quinet, Paulo Botelho e João Marcelo A. Braga; da FEEMA, Jorge P. P. Carauta; da UFRJ, Cláudia M. Vieira, e da UFRRJ, Ariane L. Peixoto, pelo auxílio nas identificações.

\section{Referências bibliográficas}

Brower, J. E. \& Zar, J. H. 1977. Field and laboratory methods for general ecology. W. C. Brown Company Publishers, Iowa.

Clark, D. B. 1996. Abolishing virginity. Journal of Tropical Ecology 12(5): 735-739.

Fundação SOS Mata Atlântica. 1998. Atlas da evolução dos remanescentes florestais e ecossistemas associados no domínio da Mata Atlântica no período 1990-1995. Fundação SOS Mata Atlântica, São Paulo.

Dean, W. 1996. A ferro e fogo. A história e a devastação da Mata Atlântica brasileira. Compania das Letras, São Paulo.

Gaston, K. J. \& Lawton, J. H. 1990. Effects of scale and habitat on the relationship between regional distribution and local abundance. Oikos 58: 329-335.
Guedes-Bruni, R. R.; Pessoa, S. V. A. \& Kurtz, B. C. 1997. Florística e estrutura do componente arbustivo-arbóreo de um trecho preservado de floresta montana na Reserva Ecológica de Macaé de Cima. Pp. 127-145. In: H. C. Lima \& R. R. GuedesBruni (eds.). Serra de Macaé de Cima: Diversidade florística e conservação em Mata Atlântica. Jardim Botânico do Rio de Janeiro, Rio de Janeiro.

Grombone, M. T.; Bernacci, L. C.; Meira Neto, J. A. A.; Tamashiro, J. Y. \& Leitão Filho, H. F. 1990. Estrutura fitossociológica da floresta semidecídua de altitude do Parque Municipal da Grota Funda (Atibaia, Estado de São Paulo). Acta Botanica Brasilica 4(2): 47-65.

Hadley, K. S. 1994. The role of disturbance, topography, and forest structure in the development of a montane forest landscape. Bulletin of the Torrey Botanical Club 121(1): 47-61.

Jarenkow, J. A. 1994. Estudo fitossociológico comparativo entre duas áreas com mata de encosta no Rio Grande do Sul. Tese de Doutorado. Universidade de São Carlos, São Carlos.

Kurtz, B. C. \& Araújo, D. S. D. 2000. Composição florística e estrutura do componente arbóreo de um trecho de Mata Atlântica na Estação Ecológica Estadual do Paraíso, Cachoeiras de Macacu, Rio de Janeiro, Brasil. Rodriguésia 51(78/79): 69-111.

Leitão-Filho, H. F. 1987. Considerações sobre a florística de florestas tropicais e sub-tropicais do Brasil. Instituto de Pesquisa de Estudos Florestais 35: 41-46.

Lieberman, M.; Lieberman, D.; Peralta, R. \& Hartshorn, G. S. 1995. Canopy closure and distribution of tropical forest tree species at La selva, Costa Rica. Journal of Tropical Ecology 11: 161-178.

Lieberman, D.; Lieberman, M.; Peralta, R. \& Hartshorn, G. S. 1996. Tropical forest structure and composition on a large-scale altitudinal gradient in Costa Rica. Journal of Ecology 84: 137-152. 
Mantovani, W. 1993. Estrutura e dinâmica da Floresta Atlântica na Juréia, Iguape, SP. Tese de Livre-Docência. Instituto de Biociência da USP, São Paulo.

Martins, F. R. 1989. Fitossociologia de florestas do Brasil: um histórico bibliográfico. Pesquisas, São Leopoldo 40: 103-164.

Martins, F. R. 1993. Estrutura de uma floresta mesófila. $2^{a}$ edição. Editora da Universidade de Campinas, Campinas.

Mazurec, A. P. 1998. Ciclagem de nutrientes em duas florestas atlânticas de encosta em diferentes altitudes na Serra do Imbé, Norte Fluminense. Dissertação de Mestrado. Centro de Biociências e Biotecnologia da UENF, Campos dos Goytacazes.

Melo, M. M. R. F. 1993. Composição florística e estrutura de trecho de Mata Atlântica de encosta, na Ilha do Cardoso (Cananéia, SP, Brasil). Dissertação de Mestrado. Instituto de Biociências da USP, São Paulo.

Melo, M. M. R. F. \& Mantovani, W. 1994. Fitossociologia de trecho de Mata Atlântica de Encosta na Ilha do Cardoso (Cananéia, SP, Brasil). Pp. 45-53. In: Anais do III Simpósio de Ecossistemas da Costa Brasileira, vol. II. ACIESP, São Paulo.

Melo, M. M. R. F.; Oliveira, R. J.; Rossi, L.; Mamede, M. C. H. \& Cordeiro, I. 1998. Fitossociologia de trecho de Mata Atlântica na Planície do Rio Verde, Estação Ecológica de Juréia, Itatins, SP, Brasil. Pp. 49-56. In: Anais do IV Simpósio de Ecossistemas Brasileiros, vol. II. ACIESP, Águas de Lindóia, SP.

Moreno, M. R.; Nascimento, M. T. \& Kurtz, B. C. 1998. Estrutura e composição florística do estrato arbóreo em duas zonas altitudinais diferentes em Mata Atlântica de encosta na Região do Imbé, RJ: Primeira aproximação. Pp.64-70. In: Anais do IV Simpósio de Ecossistemas Brasileiros, vol. II. ACIESP, Águas de Lindóia, SP.

Mori, S. A.; Boom, B. M. \& Prance, G. T. 1981. Distribution patterns and conservation of eastern Brazilian coastal forest tress species. Brittonia 33: 233-245.

Mori, S. A.; Boom, B. M.; Carvalho, A. M. \& Santo, T. S. 1983. Ecological importance of Myrtaceae in an Eastern Brasilian Wet Forest. Biotropica 15(1): 68-70.
Nascimento, M. T. \& Cunha, C. N. 1989. Estrutura e composição florística de um cambarazal no pantanal de Poconé, MT. Acta Botanica Brasilica 3(1): 45-66.

Nascimento, M. T. \& Saddi, N. 1992. Structure and floristic composition in an area of cerrado in Cuiabá, MT, Brazil. Revista Brasileira de Botânica 15(1): 47-55.

Nascimento, M. T.; Proctor, J. \& Villela, D. M. 1997. Forest structure, floristic composition and soil of na amazonian monodominant forest on Maracá Island, Roraima, Brazil. Edinburgh Journal of Botany 54(1): 1-38.

Peixoto, A. L. 1992. Vegetação da costa atlântica. Pp. 33-41. In: S. Monteiro \& L. Kaz (eds.). Floresta Atlântica. Ed. Alumbramento. Rio de Janeiro.

Pendry, C. A. \& Proctor, J. 1996. The causes of altitudinal zonation of rain forests on Bukit Belalong, Brunei. Journal of Ecology 84: 407-418.

Pessoa, S. V. A.; Guedes-Bruni, R. R. \& Kurtz, B. C. 1997. Florística e estrutura do componente arbustivo-arbóreo de um trecho secundário de floresta montana na Reserva Ecológica de Macaé de Cima. Pp. 147-167. In: H. C. Lima \& R. R. Guedes- Bruni (eds.). Serra de Macaé de Cima: Diversidade florística e conservação em Mata Atlântica. Jardim Botânico do Rio de Janeiro, Rio de Janeiro.

Proctor, J.; Lee, Y. F.; Langley, A. M.; Munro, W. R. C. \& Nelson, T. 1988. Ecological studies on Gunung Silan, a small ultrabasic mountain in Sabah, Malaysia. I. Environment, forest structure and floristics. Journal of Ecology 76: 320-340.

Rabinowitz, D. 1981. Seven forms of rarity. The biological aspects of rare plant conservation 1: 205-217.

Radam Brasil. 1983. Levantamento de recursos naturais, vol. 32, folha S/ F. 23/ 24. Rio de Janeiro/ Vitória. Ministério das Minas e Energia, Rio de Janeiro.

Richards, P. W. 1981. The tropical rain forest: an ecological study. $2^{\text {nd }}$ edition. Cambridge University Press, Cambridge.

Shepherd, G. J. 1995. FITOPAC. Manual do Usuário. UNICAMP, Campinas, SP.

Silva, A. F. 1980. Composição florística e estrutura de um trecho de Mata Atlântica de encosta no município de Ubatuba. Dissertação de Mestrado. Instituto de Biologia da UNICAMP, Campinas. 
Silva, G. C. \& Nascimento, M. T. 2001. Fitossociologia do componente arbóreo de um remanescente de mata sobre tabuleiros terciários no Norte Fluminense (mata do Carvão). Revista Brasileira de Botânica 24: 51-62.

Soares, R. O. \& Ascoly, R. B. 1970. Florestas costeiras do litoral leste (inventário florestal de reconhecimento). Brasil Florestal 1(2): 9-20.
Thomas, L. D. 1998. Floração e frutificação de algumas espécies de Mata Atlântica. Pp. 135-141. In: Anais do IV Simpósio de Ecossistemas Brasileiros, vol. II. ACIESP, Águas de Lindóia, SP.

Zar, J.H. 1996. Biostatistical analysis. $3^{\text {rd }}$ ed. PrenticeHall Inc., New Jersey. 